\title{
Commentaries
}

\section{Just how inflamed is the normal gut?}

\author{
That the science of cartography is limited \\ - and not simply by the fact that this shading of \\ forest cannot show the fragrance of balsam, \\ the gloom of cypresses, \\ is what I wish to prove
}

Eavan Boland

The mammalian intestine has evolved as a tightly regulated and intricate environment concerned with manipulating vast quantities of complex substances. Ingested matter must be processed, transported and appropriately disposed of without stimulating adverse local reactions while contaminating microorganisms with pathogenic potential must be dispatched. Cytokines are of fundamental importance in all of these processes, yet the pleiotropy, interdependence and redundancy of cytokine networks has made this a difficult and often confusing area to chart.

NORMAL INTESTINE: A SITE OF CONTROLLED INFLAMMATION Cytokine studies in the intestine have, in the past, focused on the possible role of these factors as immunological mediators of inflammatory disease states and it has generally been assumed that activation, as a result of some pathogenic process, was required for their secretion. Thus, inflammatory cytokines, including interleukin (IL) 1, IL-2, tumour necrosis factor (TNF) $\alpha$ and interferon (IFN) $\gamma$, have all been claimed to be the primary pathogenic mediators in diseases of the intestine such as Crohn's disease, ulcerative colitis and coeliac disease. ${ }^{1}$ However, the paper by Lambrechts et al (see page 643) clearly demonstrates that in the absence of stimulation, the healthy intestine contains significant numbers of IL- 4 and IFN- $\gamma$ elaborating intestinal lymphocytes. Using intracytoplasmic staining followed by flow cytometric analysis, this proportion was found to increase dramatically in response to stimulation with almost two thirds of intestinal lymphocytes expressing IFN $-\gamma$. Although this seems at first sight a surprisingly large number of differentiated, IFN- $\gamma$ secreting $\mathrm{T}$ cells, these results do correlate well with other hallmarks of a primed inflammatory immune response which characterises the healthy intestinal tract. ${ }^{2}$ These include large numbers of CD45RO expressing memory $\mathrm{T}$ cells and high expression of the activation markers CD69 and class II molecules. Thus, a majority of resident gut $\mathrm{T}$ lymphocytes seem to be terminally differentiated, activated Th1-type $\mathrm{T}$ cells primed to secrete IFN- $\gamma$. These features raise some interesting questions as to how such large numbers of potential inflammatory cells are normally kept in check. In this respect, the consistent low grade chronicity of inflammatory features found in the disease-free intestine has led to the suggestion that its normal status is "controlled inflammation". ${ }^{2}$ Thus the potential to secrete IFN- $\gamma$, the key inflammatory cytokine, must be particularly tightly regulated in vivo while remaining readily triggered. Putative key cytokines which play a role in preventing excessive inflammation would include IL-10 and transforming growth factor (TGF) $\beta$. Murine studies have suggested that reciprocal IFN- $\gamma$ and TGF- $\beta$ expression regulate the occurrence of mucosal inflammation. ${ }^{3}$ Further studies have elegantly demonstrated this by showing that, when grown in IL-10, ovalbumin specific clones of T cells express IL-10, IFN- $\gamma$ and TGF- $\beta$ but not IL- 2 or IL-4. These clones could then prevent colitis when transferred into a murine model-interestingly, only after feeding with OVA protein. ${ }^{3}$ However, caution needs to be exercised when extrapolating from mouse to man-for example, in the mouse only a small frequency of normal murine intestinal lymphocytes are differentiated to produce IFN- $\gamma{ }^{4}$ This contrasts with the human studies of Lambrechts et al where almost two thirds of intestinal lymphocytes expressed IFN- $\gamma$ when stimulated. Such strong differences suggest that different networks might operate in each species.

ADVANTAGES OF THE “CONTROLLED INFLAMMATORY” STATUS The fundamental features of the gastrointestinal tract impose a need for a range of protective mechanisms primed to deal with the potentially harmful challenges inevitably encountered during food consumption. Indeed, it is likely that this was a primary driving force for the evolution of an immune system. For humans, such mechanisms would have had distinct survival advantages when food was gathered in the wild and eaten uncooked or in unhygenic conditions. The lack of appropriate immunological stimulation as a result of eating almost sterile food in the obsessively hygienic conditions characteristic of the Western way of life may contribute to the current increase in food intolerance and associated allergies. It has already been proposed that the absence of infection may contribute to the increasing incidence of asthma and atopy. ${ }^{5}$ This recent study described an inverse association between tuberculin responses and atopic disorders in 1000 Japanese school children. It was proposed that infection, which stimulated a Th1-type environment with IL-12, IFN- $\gamma$ and TNF- $\alpha$ secretion, might protect against Th2 mediated asthma and atopy by inhibiting Th2 type cytokines. Conceivably, under modern living conditions and the absence of gastrointestinal infections, the system defaults to a predominant Th2 mode in certain individuals, thus promoting the chances of Th2 mediated diseases such as food allergy.

CYTOKINE ELABORATION AND INFLAMMATORY BOWEL DISEASE If the normal intestine is constantly in a state of controlled inflammation, it is easy to speculate how breakdown of control may contribute to the aetiopathogenesis of inflammatory bowel disease. Changes in genetic regulation of cytokine expression may significantly influence the composition of the local cytokine milieu and thus contribute to the propensity to develop inflammatory disease. There is increasing evidence that polymorphisms of the promoter regions of cytokine genes can lead to altered expression patterns and thus influence immune responses. ${ }^{6}$ Thus, a genetically influenced local environment which was rich in IFN- $\gamma$ or TNF- $\alpha$ could lead to triggering or overpriming of the system. Alternatively, low levels of IL-10 or TGF- $\beta$ could lead to breakdown in immunoregulation. Indeed, 
artificial interference with regulatory cytokine pathways using gene knockout technology has, in many cases, resulted in animal forms of chronic intestinal inflammation which resemble human inflammatory bowel disease. ${ }^{7}$ Moreover, recent studies have shown that a single infusion of a chimeric monoclonal anti-TNF antibody was an effective short term treatment in many patients with moderate to severe Crohn's disease ${ }^{8}$ However, despite such encouraging results, the complexity of cytokine networks and their overlapping roles leads one to intuit that the notion that interference with a single cytokine could cure all cases of inflammatory bowel disease may be naive and possibly dangerous in the long term.

\section{FUTURE DIRECTIONS}

Localisation of cytokine production to individual cell populations and different stages of stimulation will be fundamental to understanding how they might be ultimately manipulated in pathogenic situations. It is becoming increasingly clear that intestinal $\mathrm{T}$ cells are highly heterogeneous with regard to TCR and co-receptor expression, activation status and origin. ${ }^{9-11}$ It is likely that their cytokine profiles will be equally individual. Lambrechts $e t$ al found that virtually all IL- 4 and IFN- $\gamma$ secreting cells in the lamina propria were $\mathrm{CD} 4+$. In the epithelium, IFN- $\gamma$ elaboration was found in $\mathrm{CD} 8+$ populations as well as CD4+. Further isolation of various subpopulations of lymphocytes from both intestinal compartments and comprehensive analysis of their cytokine production potential will be required in order to understand their function. TNF, IL-12, IL-18, and TGF- $\beta$ will be particularly interesting cytokines to study from this point of view. Cytokine production by cells other than lymphocytes will also have important functional implications-for example, it already has been shown that epithelial cells elaborate IL-6, IL-10 and, more recently, IL-7. ${ }^{12}$ Development of the technical ability to analyse coexpression of two or more cytokines and changes in relative proportions of the cytokine cocktail produced by individual subpopulations will lead to more accurate mapping of cytokine networks and their influences. However, a two-dimensional fixed chart could only ever be a limited representation of this plastic, interdependent system. Some sort of a multidimensional, dynamic map will be required to make accurate predictions of how the intestinal immune response might be manipulated by influencing individual cytokine expression. Thus, computer modelling of cytokine networks may lead to successful prediction of the small changes in individual components of cytokine networks required for disease treatment and prevention while maintaining homoeostasis of the gastrointestinal immune system.

C O'FARRELLY

Education and Research Centre,

St Vincent's Hospital,

Elm Park,

Dublin 4,

Ireland

1 Sartor RB. Cytokines in intestinal inflammation: pathophysiological and clinical considerations. Gastroenterology 1994;106:533-9.

2 Johnson LR, Shanahan F. The intestinal immune system. In: Johnson LR, ed. Physiology of the gastrointestinal tract. 3rd edn. New York: Raven Press, 1994.

3 Strober W, Kelsall B, Fuss I, et al. Reciprocal IFN $\gamma$ and TGF $\beta$ responses regulate the occurrence of musosal inflammation. Immunol Today 1997;18: 61-4

4 Simpson SJ, Hollander GA, Mizoguchi E, et al. Defects in T-cell regulation. In: Kagnoff MF, Kiyono H, eds. Essentials of mucosal immunology. San Diego: Academic Press, 1996:291-304.

5 Cookson WOCM, Moffatt MF. Asthma: an epidemic in the absence of infection. Science 1997;275:41-2.

6 Wilson GA, Di Giovine FS, Duff G. Genetics of TNF $\alpha$ in autoimmunity, infection and neoplastic disease. F Inflammation 1995;45:1-12

7 Simpson, SJ, de Jong Y, Comiskey M, et al. Pathways of T cell pathology in

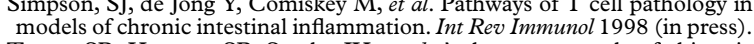

8 Targan SR, Hanauer SB, Sander JH, et al. A short term study of chimeric monoclonal antibody cA2 to tumour necrosis factor for Crohn's disease. $N$ Engl f Med 1997;337:1029-35.

9 Kagnoff M. Mucosal immunology: New frontiers. Immunol Today 1996;17: $57-9$

10 Lynch S, Kelleher D, Feighery C, et al. Flow cytometric analysis of intraepithelial lymphocytes from human small intestinal biopsies reveals populations of CD4+CD8+ and CD8 $\alpha \alpha+$ cells. Eur $\mathcal{F}$ Gastroenterol Hepatol 1993; 5:907-12

11 Carton J, Abuzakouk A, Feighery C, et al. Significant populations of unconventional $\mathrm{T}$ lymphocytes(CD4+CD8+ and $\mathrm{CD} \alpha+\beta-$ ) in human diseasefree small intestinal lamina propria. Eur $\mathcal{f}$ Gastroenterol Hepatol 1998 (in free small
press).

12 Madrigal Estebas L, McManus R, Byrne B, et al. Human small intestinal epithelial cells secrete interleukin-7 and differentially express two different interleukin-7 mRNA transcripts: Implications for extrathymic T cell differentiation. Hum Immunol 1997;58:83-90.

\section{Intestinal metaplasia and the squamocolumnar junction: what does it all mean?}

In theory, the diagnosis of Barrett's oesophagus should be fairly simple, namely the presence of intestinal metaplasia anywhere in the tubular oesophagus. However, recognising where the tubular oesophagus ends and the saccular stomach begins is fraught with difficulty. The precise junction may be difficult to identify endoscopically because of the presence of a hiatus hernia, inflammation and some disassociation between the squamocolumnar junction and the lower oesophageal sphincter zone in normal individuals. Histologically, the squamous mucosa of the oesophagus accurately defines this area, but distinguishing normal gastric cardia and metaplastic changes of the distal oesophagus may be difficult. Normally, the mucosa of the cardia is composed of tightly packed mucus secreting glands with a lamina propria devoid of any significant inflammatory component. On occasion, biopsy specimens from the cardia show an increase in the amount of acute and chronic inflammatory cells, a condition termed carditis. The cardia can also have associated areas of intestinal metaplasia with goblet cells, histologically similar to the well described intestinal metaplasia in the distal stomach or classically defined as Barrett's oesophagus when located in the tubular oesophagus. Finally, structures just do not sit still in the oesophagus during endoscopy, making precise location and biopsy even more difficult. Thus, it is difficult to know in every case whether the intestinal metaplasia is from the distal oesophagus or from the cardia.

The classic definition of Barrett's oesophagus requiring the presence of at least $2-3 \mathrm{~cm}$ of intestinal metaplasia above the oesophagogastric junction, protected us from some of these difficulties. However, the evolving concept of short segment Barrett's oesophagus, namely specialised columnar epithelium lining less than $2-3 \mathrm{~cm}$ of the distal oesophagus, now raises the question as to where to obtain biopsy specimens in patients undergoing upper endoscopy. This controversy was further intensified in 1994 after Spechler and colleagues published their study of consecutive patients 
referred for elective upper endoscopy for a variety of upper gastrointestinal symptoms. ${ }^{1}$ All patients had two biopsy specimens taken from the columnar side of the oesophagogastric junction, even if there was $3 \mathrm{~cm}$ or less of endoscopically apparent Barrett's epithelium. Of the 142 patients studied, two ( $1 \%$ ) had endoscopic evidence of Barrett's oesophagus, whereas $26(18 \%)$ had intestinal metaplasia below a normal appearing squamocolumnar junction. The groups did not differ significantly in the frequency of symptoms and endoscopic signs of gastrooesophageal reflux. However, patients with the specialised columnar epithelium at the normal oesophagogastric junction were predominantly white men, the same demographic pattern as seen in conventional length Barrett's oesophagus. Subsequently, several groups have identified short segment Barrett's oesophagus in $8-10 \%$ of almost 500 consecutive patients undergoing upper endoscopy in various settings. ${ }^{2-4}$ Male sex usually predominates and reflux symptoms are common. The study by Dias Pereira et $a l$ in this issue (see page 659) confirms these observations while suggesting that the prevalence of short segment Barrett's oesophagus does not increase with advancing age, an observation somewhat different from that in classic Barrett's oesophagus.

Could all of these simply be intestinal metaplasia of the gastric cardia? The answer is still evolving but several issues were tackled in recent studies. How common is intestinal metaplasia of the cardia? In an unselected group of patients undergoing endoscopy in a Veteran's Affairs Hospital, Morales and colleagues ${ }^{3}$ found intestinal metaplasia of the cardia in $24(23 \%)$ of 104 patients, a prevalence similar to the $25 \%$ reported by Dias Pereira and colleagues. Others report similar findings but with a lower prevalence. Hirota and coworkers ${ }^{5}$ found intestinal metaplasia of the cardia in only $47(5.3 \%)$ of 889 patients, whereas in our recent study of 86 patients undergoing elective endoscopy, we found intestinal metaplasia of the cardia in $9 \% .^{6}$ What are the demographics of patients with intestinal metaplasia the cardia? Men predominate in some populations, but others find that women are common. Most studies find a lower frequency of reflux symptoms. In fact, only two of eight patients with intestinal metaplasia of the cardia in our study had reflux symptoms. ${ }^{6}$ Finally, most studies find intestinal metaplasia of the cardia predominantly in an older population. Are there any histological clues to the aetiology of intestinal metaplasia of the cardia? Nearly all patients with intestinal metaplasia of the cardia have associated carditis. Despite an intriguing hypothesis that carditis is an inflammatory change that occurs as a consequence of gastro-oesophageal reflux disease, ${ }^{7}$ this seems not to be the case. For example, we found carditis in $41 \%$ of our control population compared with $40 \%$ of patients with reflux. ${ }^{6}$ Rather, Helicobacter pylori infection seems be the cause of most cases of carditis and associated intestinal metaplasia of the cardia. Morales et al found that $68 \%$ of patients with intestinal metaplasia of the cardia were infected with this organism. ${ }^{3}$ Likewise, we found that intestinal metaplasia of the cardia was more common among controls $(22 \%)$ than patients with reflux (3\%). All eight of these patients has associated carditis, seven with biopsy and/or serological evidence of $H$ pylori infection, and six had intestinal metaplasia elsewhere in the stomach. What is the association between Barrett's oesophagus and intestinal metaplasia of the cardia? Morales and colleagues ${ }^{3}$ found intestinal metaplasia of the cardia in two (18\%) of 11 patients with Barrett's oesophagus. Weston and colleagues ${ }^{8}$ found intestinal metaplasia of the cardia in eight $(7 \%)$ of 119 patients with Barrett's epithelium. Interestingly, it was encountered in six $(10 \%)$ of 59 patients with short segment Barrett's oesophagus and only two (3.3\%) of 60 patients with $>2 \mathrm{~cm}$ of Barrett's epithelium. Thus, short segment Barrett's oesophagus and intestinal metaplasia of the gastric cardia may overlap and may be an artefact of the true site of biopsy at the oesophagogastric junction.

What does this all mean? At the time of endoscopy, landmarks should be carefully defined and if the squamocolumnar junction is above the level of the oesophagogastric junction, biopsy samples should be obtained. Helpful findings for short segment Barrett's oesophagus include a straight and regular squamocolumnar junction displaced upwards in relation to the gastro-oesophageal junction by $<3 \mathrm{~cm}$ or an irregular squamocolumnar junction with eccentric tongues of red mucosa extending above this junction. If intestinal metaplasia is present, the patient is considered to have Barrett's oesophagus and should be placed on an endoscopic surveillance programme as dysplasia and adenocarcinoma may develop in these patients. In clinical practice, biopsy samples should not be taken routinely below a normal appearing squamocolumnar junction. Goblet cells found at this level are probably secondary to intestinal metaplasia of the gastric cardia, probably resulting from chronic $H$ pylori infection. This can be confirmed by serological studies for $H$ pylori as well as biopsy of the more distal antrum and body. The implications for endoscopic surveillance in patients with intestinal metaplasia of the cardia is unknown at this time. However, it is intriguing to speculate that the increase in adenocarcinoma at the oesophagogastric junction may be secondary to two processes. That is, an increased prevalence of Barrett's oesophagus in an aging white male population as well as an increase in the prevalence of multifocal atrophic gastritis and intestinal metaplasia which can include the cardia secondary to $H$ pylori infection in aging individuals, both male and female.

Chairman and Professor of Medicine,

J E RICHTER

Department of Gastroenterology,

The Cleveland Clinic Foundation,

9500 Euclid Avenue, S40,

Cleveland, Ohio 44195, USA

1 Spechler SJ, Zeroogian JM, Antonioli DA, et al. Prevalence of metaplasia at the gastro-oesophageal junction. Lancet 1994;344:1533-6.

2 Johnston MH, Nammond AS, Laskin W, et al. The prevalence and clinical characteristics of short segments of specialized intestinal metaplasia in the distal esophagus. Am ₹ G Gastroenterol 1996;91:1507-11.

3 Morales TG, Sampliner RE, Bhattacharyya T. Intestinal metaplasia of the cardia. Am f Gastroenterol 1997;92:414-18.

4 Chalasani N, Wo JM, Hunter JG, et al. Significance of intestinal metaplasia in different areas of the esophagus including esophagogastric junction. Dig Dis Sci 1997;42:603-7.

5 Hirota WK, Loughney TM, Lanzas DJ, et al. Is Helicobacter pylori associated with specialized intestinal metaplasia of the esophagus or stomach? A prospective study of 889 patients [abstract]. Gastroenterology 1997;112: A149.

6 Goldblum JR, Vicar JJ, Falk GF, et al. Inflammation and intestinal metaplasia of the gastric cardia: the role of gastroesophageal reflux and H. pylori infection. Gastroenterology 1998 (in press).

7 Oberg S, Peters JH, De Meester TR, et al. Inflammation and specialized intestinal metaplasia of cardiac mucosa is a manifestation of gastroesophaintestinal metaplasia of cardiac mucosa is a man.
geal reflux disease. Ann Surg 1997;226:522-32.

geal reflux disease. Ann Surg 1997;226:522-32.
8 Weston AP, Krmpotich P, Cherian R, et al. Prospective evaluation of intestinal metaplasia and dysplasia within the cardia of patients with Barrett's esophagus. Dig Dis Sci 1997;42:597-602. 


\section{Shock news for the gut}

In a variety of pathological states, notably ischaemiareperfusion injury, organ infarction, and shock, there are substantial increases in the normally low circulating concentrations of endothelin-1 (ET-1). ${ }^{12}$ This is more than just a symptom. ET-1 seems to be an active participant in these disorders as antibodies directed against ET-1 and endothelin receptor antagonists are protective. ${ }^{12}$ Interestingly, although studies in isolated cells suggest that the production of ET-1 can only be up-regulated over the course of hours, increases in vivo can be recorded within much shorter periods. For example, intravenous injection of a high dose of endotoxin to anaesthetised rats causes within 5-10 minutes both a dramatic fall in blood pressure and a notable increase in haematocrit. Both of these effects are greatly reduced by treatment with endothelin receptor antagonists active at the $\mathrm{ET}_{\mathrm{A}}$ receptor. ${ }^{3}$ Clearly, the speeds of these responses suggest a direct effect of high dose endotoxin. When given in lower doses endotoxin causes increases in plasma ET-1 over periods of hours rather than minutes. These more delayed responses are indirect effects of endotoxin and are secondary to the release of cytokines, particularly tumour necrosis factor (TNF) $\alpha$ and interleukin-1 $\beta$. Increases in circulating ET-1 caused by endotoxin in both pigs and baboons are noticeably reduced by treatment with anti-TNF- $\alpha .^{45}$ Similarly, and more importantly, in human septic shock there is a clear positive correlation between the circulating concentrations of TNF- $\alpha$ and ET- $1 .{ }^{6}$

What, however, are the clinical implications of either direct or cytokine mediated increases in ET-1 caused by endotoxin? In this issue Oldner et al (see page 696) report that in a porcine model of endotoxaemia, administration of bosentan (a non-selective endothelin $\mathrm{ET}_{\mathrm{A} / \mathrm{B}}$ receptor antagonist) restores gut oxygen delivery and reverses intestinal mucosal acidosis. Clearly, these are beneficial effects, but would endothelin receptor antagonists be useful in human endotoxaemia? In their model Oldner et al follow the course of changes in anaesthetised pigs over five hours following endotoxin infusion. Endotoxin causes an early fall in blood pressure and an accompanying rise in blood endothelin concentrations associated with the immediate effects of endotoxin discussed earlier. Over the next few hours the continued fall in blood pressure is unaffected by bosentan. However, bosentan lowers systemic vascular resistance and so improves tissue perfusion. Within the gut these beneficial effects of bosentan are evident as support of portal blood flow, increase in portal venous oxygen saturation and gut oxygen delivery, and prevention of the fall in gastrointestinal mucosal $\mathrm{pH}$. These outcomes seem to indicate that endothelin antagonists would be therapeutically beneficial in human endotoxic shock. However, we should be cautious in drawing such conclusions. In particular the short experimental period chosen by Oldner et al permits study of the initial responses to endotoxaemia, but not the longer term responses that are more relevant to clinical practice. More prolonged exposure to endotoxin leads to many profound effects. These effects follow alterations in the cellular expressions of proteins and so take hours to become apparent. In particular, recent research efforts have examined how excessive nitric oxide, produced by newly synthesised inducible nitric oxide synthase, underlies the vascular hyporesponsiveness found after longer periods of endotoxaemia. At these latter time points much clinical effort is aimed at supporting blood pressure. ${ }^{7}$ The administration of an agent such as bosentan, which antagonises the pressor effects of endogenously produced ET-1, may precipitate a further critical fall in blood pressure. Indeed, in the rat endothelin receptor antagonists potentiate endotoxaemia associated hypotension ${ }^{8}$ and increase mortality. ${ }^{9}$

Weighing up the current evidence leads us to the conclusion that Oldner et al are correct in suggesting that endothelin receptor antagonists would prove useful in reducing gut ischaemia in human septic shock. This could be of benefit as gut mucosal hypoperfusion is well known to be associated with poor prognosis. ${ }^{10}$ However, the administration of endothelin receptor antagonists to humans with established sepsis may well produce dangerous falls in blood pressure that could more than offset any benefits on gut perfusion.

T D WARNER

$S$ A MCCARTNEY

Vascular Inflammation,

The William Harvey Research Institute,

St Bartholomew's and the Royal London

School of Medicine and Dentistry,

Charterhouse Square,

London EC1M 6BQ, UK

1 Rubanyi GM, Polokoff MA. Endothelins: molecular biology, biochemistry, pharmacology, physiology and pathophysiology. Pharmacol Rev 1994;46:325-415.

2 Warner TD, Battistini B, Doherty AM, et al. Endothelin receptor antagonists: actions and rationale for their development. Biochem Pharmacol 1994;48:625-35.

3 Allcock GH, Warner TD. Activation of ETA receptors is partially responsible for the rapid increase in haematocrit induced by bacterial lipopolysaccharide in the rat. Life Sci 1997;60:PL271-6.

$4 \mathrm{Han}$ JJ, Windsor A, Drenning DH, et al. Release of endothelin in relation to tumor necrosis factor- $\alpha$ in porcine Pseudomonas aeruginosa-induced septic shock. Shock 1994;1:343-6.

5 Redl H, Schlag G, Bahrami S, et al. Big-endothelin release in baboon bacteremia is partially TNF dependent. F Lab Clin Med 1994;124:796801 .

6 Takakuwa T, Endo S, Nakae H, et al. Plasma levels of TNF- $\alpha$, endothelin 1 and thrombomodulin in patients with sepsis. Res Commun Chem Pathol Pharmacol 1994;84:261-9.

7 Kilbourn RG, Szabo C, Traber DL. Beneficial versus detrimental effects of nitric oxide synthase inhibitors in circulatory shock: lessons learned from experimental and clinical studies. Shock 1997;7:235-46.

8 Gardiner SM, Kemp PA, March JE, et al. Enhancement of the hypotensive and vasodilator effects of endotoxaemia in conscious rats by the endothelin antagonist, SB 209670. Br f Pharmacol 1995;116:1718-19.

9 Ruetten H, Thiemermann C, Vane JR. Effects of endothelin receptor antagonist, SB209670, on circulatory failure and organ injury in endotoxic shock in the anaesthetized rat. Br F Pharmacol 1996;118:198-204.

10 Mythen MG, Webb AR. Intra-operative gut mucosal hypoperfusion is associated with increased post-operative complications and cost. Int Care Med 1994;20:99-104. 